\title{
STRAIN RELAXATION OF ZnTe/CdTe AND CdTe/ZnTe HETEROSTRUCTURES: IN SITU STUDY*
}

\author{
F. RIESZ \\ Research Institute for Technical Physics of the Hungarian Academy of Sciences \\ P.O. Box 76, 1325 Budapest, Hungary \\ S. Kret, G. Karczewski, T. WoJtowicz and J. KossuT \\ Institute of Physics, Polish Academy of Sciences \\ Al. Lotników 32/46, 02-668 Warsaw, Poland
}

The strain relaxation kinetics of $\mathrm{ZnTe} / \mathrm{CdTe}$ and $\mathrm{CdTe} / \mathrm{ZnTe}$ heterostructures grown on GaAs substrates by molecular beam epitaxy are studied by in situ reflection high-energy electron diffraction. The observed critical layer thickness is 5 monolayers for $\mathrm{ZnTe} / \mathrm{CdTe}$ and less than 1 monolayer for $\mathrm{CdTe} / \mathrm{ZnTe}$. The relaxation is anisotropic. Dislocation core parameters and relaxation rate constants were determined using a kinetic model and assuming strain-dependent activation energy of dislocation movement.

PACS numbers: $61.14 . \mathrm{Hg}, 68.55 . \mathrm{Bd}, 68.65 . \mathrm{tg}$

The strain relaxation mechanism in lattice-mismatched heterostructures is of high interest from the point of view of both fundamentals and practical applications. Reflection high-energy electron diffraction (RHEED), a built-in facility of all molecular beam epitaxy (MBE) systems, gives a powerful in situ tool for the study of the relaxation kinetics.

In this paper we report on an in situ RHEED study of the relaxation kinetics of MBE-grown ZnTe/CdTe and CdTe/ZnTe heterostructures. For details of the growth, see Ref. [1]. Following a few monolayers (MLs) ZnTe, a $2 \mu \mathrm{m}$ CdTe buffer was grown first on a (100) GaAs substrate miscut towards [010] by $2^{\circ}$, followed by alternating multilayers of about $400 \mathrm{~nm} \mathrm{ZnTe}$ and CdTe. This thickness was large enough to yield completely relaxed buffers for the subsequent growth. The growth rate was typically $g=0.65-0.9 \mathrm{ML} / \mathrm{s}$, as monitored by the RHEED intensity oscillations. The growth temperature was $300^{\circ} \mathrm{C}$. Beam equivalent pressure ratios were $\mathrm{Zn} / \mathrm{Te}=0.4$ and $\mathrm{Cd} / \mathrm{Te} \approx 1.1$. The growth was always started on a

*This work was supported, in part, by the (Hungarian) National Scientific Research Fund (OTKA) through grant F 016278 (Budapest) and by the Committee for Scientific Research (Poland) under grant 8T11B02108 (Warsaw). 
Te-stabilized surface. The lattice parameter variation was extracted in real time from the distance of the 01 and $0 \overline{1}$ streaks of the RHEED pattern using a CCD camera, a video recorder and a dedicated software [1]. Experiments were carried out using both [011] and [011] e-beam directions to reveal the anisotropy of the relaxation process; these directions probe the lattice spacings of (011) and (011) planes, respectively. Separate growth sequences with the same parameters were used to study the orthogonal directions. To determine the lattice misfit $f$, the substrate or buffer layer's lattice parameter was used as a reference.

The experimental data are interpreted using a kinetic model [2] which is based on the Dodson-Tsao model [3]. This model correctly includes multiplication of misfit dislocations and the anisotropy of relaxation, and allows for a more realistic correspondence of model parameters to real physical entities. The time evolution of the linear misfit dislocation density $\rho_{\mathrm{L}}$ in a given $\langle 011\rangle$ direction is

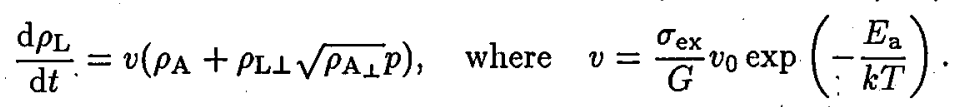

Here $\rho_{\mathrm{A}}$ is the substrate (or buffer layer) threading dislocation (or other source) density, $v$ is the dislocation velocity, $v_{0}$ is a constant, $E_{\mathrm{a}}$ is the activation energy for dislocation glide or climb and $G$ is the shear modulus; $\rho_{\mathrm{L}} \sqrt{\rho_{\mathrm{A}}} p$ is the multiplication term, $p$ being the multiplication probability at a dislocation intersection. The subscript $\perp$ refers to the other (orthogonal) $\varnothing$ direction. The driving force to relaxation is the excess stress $\sigma_{\mathrm{ex}}[2,3]$, which, for glide, is given by

$$
\begin{aligned}
\sigma_{\mathrm{ex}} & =\frac{2 G}{1-\nu} \cos \varphi\left\{\cos \lambda\left[(f-\gamma)+\nu\left(f-\gamma_{\perp}\right)\right]\right. \\
& \left.-\frac{1-\nu \cos ^{2} \alpha}{4 \pi(h / b)}\left(\ln \frac{\omega h}{b}+1\right)\right\} .
\end{aligned}
$$

Here $\nu$ is the Poisson ratio, $\gamma$ is the relaxed part of strain, $\lambda$ is the angle between the Burgers vector and that line in the epilayer plane which is perpendicular to the glide plane/surface intersection, $\varphi$ is the angle between the slip plane normal and the surface, $\alpha$ is the angle between the Burgers and line vectors, $b$ is the length of the Burgers vector and $\omega$ is the dislocation core parameter. For climb, a similar formula can be used, but $\cos \varphi$ should be replaced by $\sin \varphi$ since climb is perpendicular to the glide plane. The instantaneous film thickness $h$ is given by $h=g t$. The relieved strain is then $\gamma=\rho_{\mathrm{L}} \mathrm{b} \cos \lambda$. The equilibrium critical thickness occurs at $\sigma_{\mathrm{ex}}=0$; this condition gives also the equilibrium strain at a given thickness.

In all cases, the RHEED patterns were sharp, indicating a perfect layer-by-layer growth. The RHEED curves show an abrupt coherency breakdown. The observed critical thickness for $\mathrm{ZnTe} / \mathrm{CdTe}$ is about $5 \mathrm{MLs}$ in both $\langle 011\rangle$ directions, in agreement with other RHEED studies [4]. The fit of $\sigma_{\mathrm{ex}}=0$ to this value yields a core parameter of $\omega=3$ and 0.7 for pure edge and $60^{\circ}$ misfit dislocations, respectively. (Lattice parameters of $0.6481 \mathrm{~nm}$ and $0.6105 \mathrm{~nm}$, and the Poisson ratios of 0.41 and 0.36 were used for $\mathrm{CdTe}$ and $\mathrm{ZnTe}$, respectively, in the fits.)

Figure 1 shows the experimental relaxation curves in both $\langle 011\rangle$ directions, the equilibrium strain curves from $\sigma_{\mathrm{ex}}=0$ for edge and $60^{\circ}$ dislocations as calculated using the above core parameters, and other fit curves, as detailed below. The 


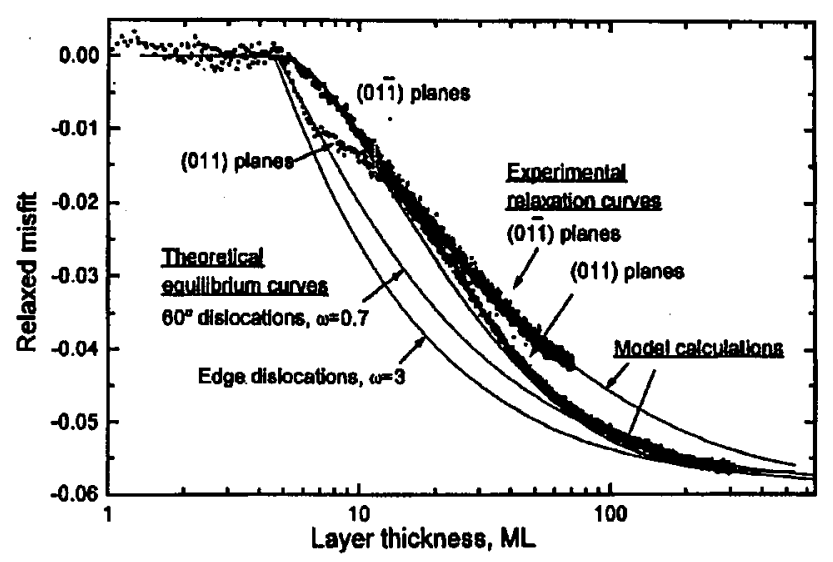

Fig. 1. Relaxation curves for $\mathrm{ZnTe} / \mathrm{CdTe}$ with the equilibrium strain curves for edge and $60^{\circ}$ dislocations and model calculations. The total misfit is $f=-0.058$. For details of the fits, see text.

close-to-equilibrium nature of the relaxation at this low temperature is probably due to the high dislocation velocity characteristic of II-VI semiconductors. Based on transmission electron microscopic studies by other groups [4], we assume edge dislocations in our fits. Only poor fits can be obtained using our model; however, if we assume an Arrhenius-type dependence of the relaxation rate constant $v \rho_{\mathrm{A}}$ on strain yielding $v \rho_{\mathrm{A}} \propto \exp (-\gamma c)$, where $c$ is a constant, almost perfect fits are obtained. (Inclusion of multiplication gave even worse fits.) This assumption is justified physically, since strain increases lattice spacings promoting dislocation movement. The fits shown in Fig. 1 were obtained by $v \rho_{\mathrm{A}}(\gamma=0)=10^{8} \mathrm{~s}^{-1} \mathrm{~m}^{-1}$ (in both directions) and $c(01 \overline{1})=58$ and $c(011)=37$. This anisotropy might be connected to the anisotropic surface structure [4]. In addition, full relaxation of the misfit strain is observed.

The critical thickness of $\mathrm{CdTe} / \mathrm{ZnTe}$ is very small ( $<1 \mathrm{ML}$ ) (Fig. 2), in contrast to previous reports [5] (5 MLs was found by RHEED and other methods). A reasonable fit to this value can be made by taking $\omega=1$ for edge dislocations; no fit is possible for $60^{\circ}$ dislocations. The equilibrium strain curve is shown in Fig. 2 for comparison, assuming total relaxation in the [011] direction. Surprisingly, the relaxation is faster in the [011] direction than predicted by the equilibrium model. The anisotropy is just reserved with respect to $\mathrm{ZnTe} / \mathrm{CdTe}$; this might be connected with the reversal of the strain sign or the different flux ratio. Again, nearly full relaxation of misfit strain is found. The strong deviation from the model reveals the limits of continuum models at the monolayer scale. The operation of additional relaxation mechanisms (e.g. stacking faults) cannot be ruled out as well.

In conclusion, we analyzed the strain relaxation behavior of $\mathrm{ZnTe} / \mathrm{CdTe}$ and $\mathrm{CdTe} / \mathrm{ZnTe}$ heterostructures by RHEED. An anisotropy of the relaxation was observed. Dislocation core parameters and relaxation rate constants were determined using a kinetic model assuming strain dependent activation energy of 


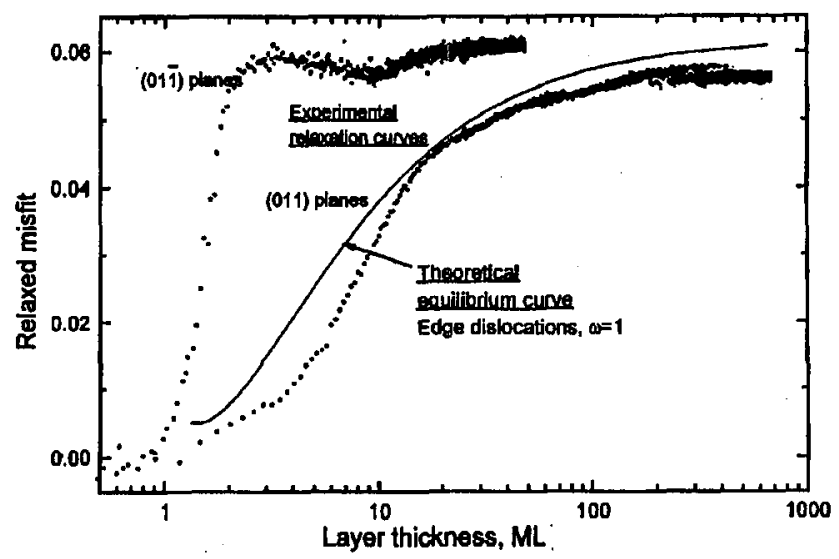

Fig. 2. Relaxation curves for $\mathrm{CdTe} / \mathrm{ZnTe}$ and the equilibrium strain curve for edge dislocations. The total misfit is $f=0.0616$.

dislocation movement for $\mathrm{ZnTe} / \mathrm{CdTe}$; strong deviation from the model was found for $\mathrm{CdTe} / \mathrm{ZnTe}$.

\section{References}

[1] S. Kret, G. Karczewski, A. Zakrzewski, P. Dłużewski, A. Dubon, T. Wojtowicz, J. Kossut, C. Delamarre, J.Y. Laval, Acta Phys. Pol. A 88, 795 (1995).

[2] F. Riesz, in preparation.

[3] B.W. Dodson, J.Y. Tsao, Appl. Phys. Lett.51, 1325 (1987); Erratum 52, 852 (1988).

[4] J. Eymery, S. Tatarenko, N. Bouchet, K. Saminadayar, Appl. Phys. Lett. 64, 3631 (1994).

[5] J. Cibert, Y. Gobil, L.S. Dang, S. Tatarenko, G. Feuillet, P.H. Jouneau, K. Saminadayar, Appl. Phys. Lett. 56, 292 (1990). 